\title{
Imaging to study solid tumour origin and progression: lessons from research and clinical oncology
}

\author{
Stefania Raimondo and Giovanni Zito
}

Biomedical imaging in recent decades has clarified our understanding of normal and pathological cellular processes in vivo. In particular, this approach recently provided insights into processes occurring at a molecular or genetic level rather than at the anatomical level. The evolution of this discipline by engineering have led to its integration into biomedical research to (1) increase sensitivity and resolution imaging and to (2) improve tissue and cell specificity. Currently, imaging approaches are used in three different biomedical areas: (a) identification of cellular processes in physiological and disease state; (b) in vivo single-cell imaging; and (c) identification of new prognostic and therapeutical strategies. In this review, we will focus on the state of art of biomedical imaging in cancer. Specifically, we will highlight the most important advances in imaging tools available for basic and translational cancer research, with a particular emphasis on solid tissue malignancies.

Immunology and Cell Biology advance online publication, 4 April 2017; doi:10.1038/icb.2017.17

\section{INTRODUCTION}

Cancer is a leading cause of death worldwide, accounting for millions of deaths every year. ${ }^{1}$ Although several steps forwards have been made in recent years to understand the biological processes involved in the development of this disease, basic and translational research is still far away from a definitive cure. This is driven by tumour heterogeneity within patients, thus making it difficult to define a unique type of treatment for the different type of cancers. Epidemiological studies recently demonstrated that solid tumours accounted for approximately $80 \%$ of cancer deaths. ${ }^{2}$ In particular, epithelial cancers include breast, lung, colon, prostate and ovarian cancers. Recently, several studies changed the way we visualise cancer development from normal tissues; we learned indeed that tumour initiation and growth is controlled by a subpopulation of cells that maintain stem cell-like features. These cells, named cancer stem cells (CSCs) or tumour-initiating cells, represent the current target of the pharmacological treatments, as their eradication might be essential for long-lasting remission and cure of cancer. $^{3}$

Along with the characterisation of CSCs or tumour-initiating cells, tremendous steps forward have been made in the identification of the tumour microenvironment cellular composition and its role in cancer progression, metastasization and resistance to therapy. ${ }^{4}$

These progresses would not have been possible without advances in biomedical imaging, as they provide the necessary tools to label and follow cell clones over time in physiological and disease states, as well as define the interactions between tumour cells and their niche. ${ }^{5}$ In the first part of this review, we will highlight the imaging techniques mainly used in cancer research and their contribution to the field, with particular emphasis on (1) the identification of the cellular origin of solid tumours and (2) the cellular and molecular characterisation of tumour cell/microenvironment interaction. We will then visit how these imaging tools have influenced clinical oncology.

\section{BIOMEDICAL IMAGING, SOLID TUMOURS AND CANCER STEM} CELL (CSCS) IN BASIC RESEARCH

Recent advances in engineering science led to the development of several types of imaging tools that can be applied in vivo to study cancer initiation, progression and metastasis. ${ }^{5}$ In basic research, imaging approaches are mostly used to define cellular and molecular aspects of tumour biology that could not be observed otherwise. However, it is important to mention that every imaging modality has its value depending on the purpose of the study. For example, optical imaging is mainly used to study tumour origin, propagation and tumour/microenvironment interaction, while positron emission tomography (PET) or magnetic resonance imaging (MRI) are mostly applied to define therapeutic response and metastatic dissemination (Figure 1). For the purpose of this review, optical imaging modalities will be analysed and discussed for basic cancer research studies, while MRI, PET and others will be discussed later to describe their use in clinical cancer management.

\section{OPTICAL IMAGING}

Fluorescence imaging to trace tumour cell of origin

In order to study CSCs and their behaviours in microenvironments, researchers have mostly relied on fluorescence imaging. This approach is crucial as the technique is easily accessible and allows (1) analysis of CSCs at a single-cell resolution and (2) accurate phenotyping of CSC within the tumour by simultaneously combining multiple 


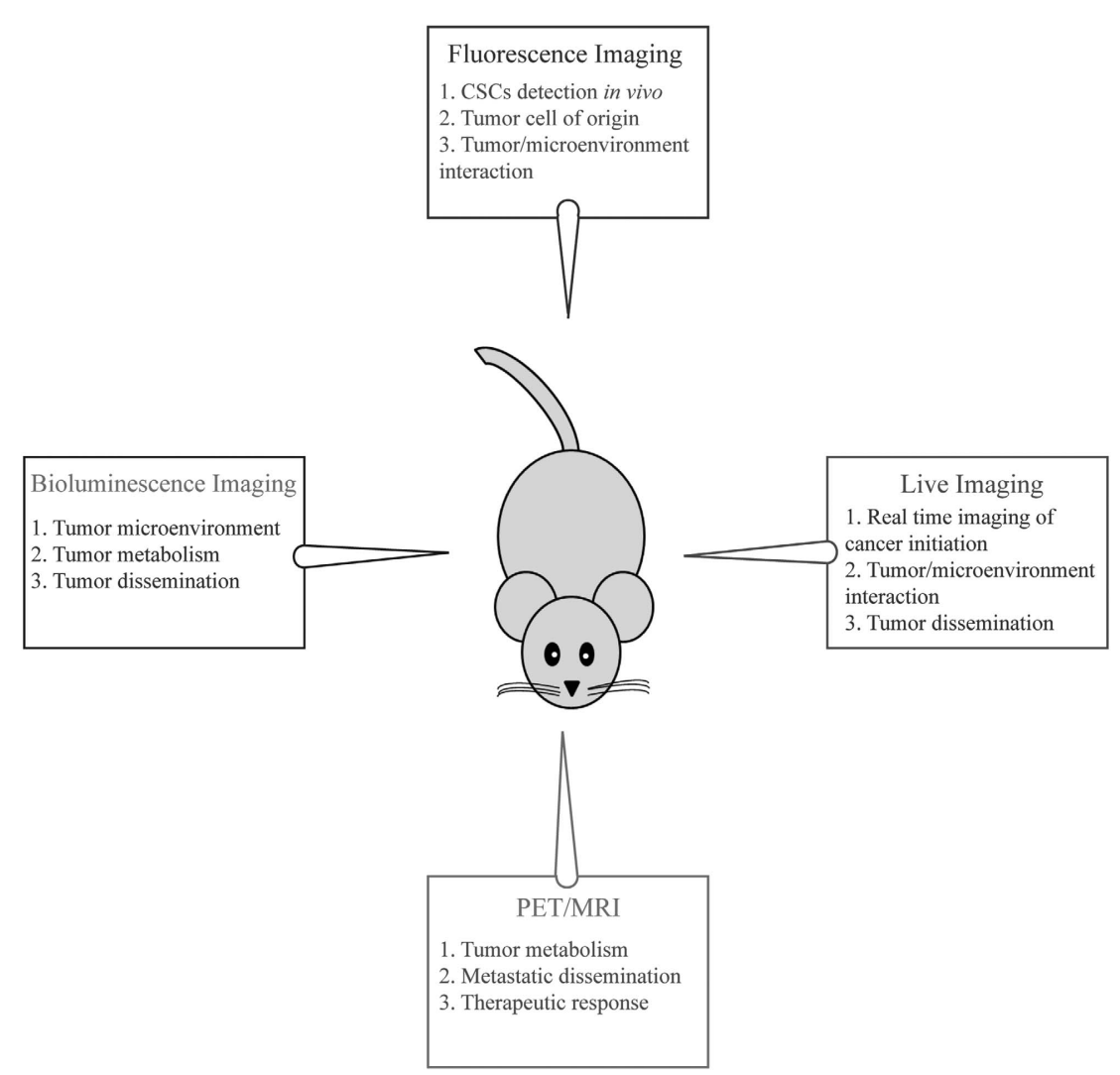

Figure 1 Schematic representation of optical imaging applications in basic cancer research. A full colour version of this figure is available at the Immunology and Cell Biology journal online.

fluorophores. ${ }^{6}$ Fluorescence imaging requires the use of fluorophores and fluorescent proteins excited by an external light source. For studies in solid epithelial tumours, fluorescence imaging was initially used in vivo to detect CSCs, based on monoclonal labelled antibodies against the well-known CSC marker CD133. ${ }^{7}$ By combining this information with additional surface markers, CSC activity can be traced in vivo in diverse contexts. For instance, Vlashi et al. ${ }^{8}$ have developed a proteasome reporter system to track CSCs in mammary tumours. By engineering a breast cancer cell lines to stably express a fluorescent fusion protein (ZsGreen-ornithine decarboxylase) that accumulates in the cells with reduced proteasome activity, they demonstrated that $\mathrm{ZsGreen}^{+}$cells were bona fide CSCs, thus providing new tools that can be used to monitor CSC activity in an in vivo tumour model.

Our knowledge of CSCs function has been expanded in solid tumours by incorporating lineage-tracing techniques, thus providing important information on the cellular origin of epithelial tumours. One example is the Cre-loxP-based genetic lineage-tracing system utilised in tandem with conditional expression of a reporter gene (that is, fluorescent protein or $\beta$-galactosidase) in a restricted cell lineage and in its progeny (Figure 2). ${ }^{9}$ By these means, all labelled cells can be traced spatially and temporally, allowing their role during development, tissue repair and in a disease state to be accured defined. ${ }^{10,11}$ By using the lineage-tracing system, in tandem with conditional expression/inhibition of disease-specific oncogenes/oncosuppressors several groups have now identified the cell of origin in solid tumours, including skin, lung and colon (discussed below). ${ }^{12}$
Using this approach, the cellular origin of basal cell carcinomas, squamous cell carcinoma and keratoacanthoma have now been defined. Youssef et al. ${ }^{13}$ demonstrated that Sonic Hedgehog constitutive signalling activation in the long-lived stem cells residing in the interfollicular epidermis gave rise to basal cell carcinomas. In addition, two groups have showed that p53 deletion, along with $\mathrm{Kras}^{\mathrm{G} 12 \mathrm{D}}$ overexpression in the hair follicle stem cells, is responsible for squamous cell carcinoma formation. ${ }^{14,15}$ Finally, by using fluorescent GFP-reporters, we demonstrated that hair follicle stem cells contributed to keratoacanthoma formation. ${ }^{16}$

Different studies combining lineage-tracing, reporter genes and oncogene/oncosuppressor activation/deletion, defined the cell of origin of lung cancers. In particular, Bern et al. ${ }^{17}$ demonstrated the heterogeneous derivation of small cell lung cancer; specifically, by using different lineage promoters, the authors demonstrated that small cell lung cancer might derive from cells of both the neuroendocrine and the alveolar type II lineage.

Finally, Barker and collaborators demonstrated that crypt stem cells in the intestine gave rise to colon cancer. In particular, by labelling with GFP the intestinal Lgr5 ${ }^{+}$stem cells, combined with APC deletion, they showed that Wnt activation in the slow-cycling stem cells contributed to adenoma formation. ${ }^{18}$ The same authors elegantly expanded this hypothesis, by using a novel multicolour Cre reporter (Rosa26R-Confetti), and demonstrated that $\operatorname{Lgr}^{+}$stem cells constantly fuelled the growth of such tumours. ${ }^{19}$

Thus, the literature above illustrated the power of fluorescence imaging to understand cancer origin and molecular pathways that 


\section{Genetic Lineage Tracing System in mice}
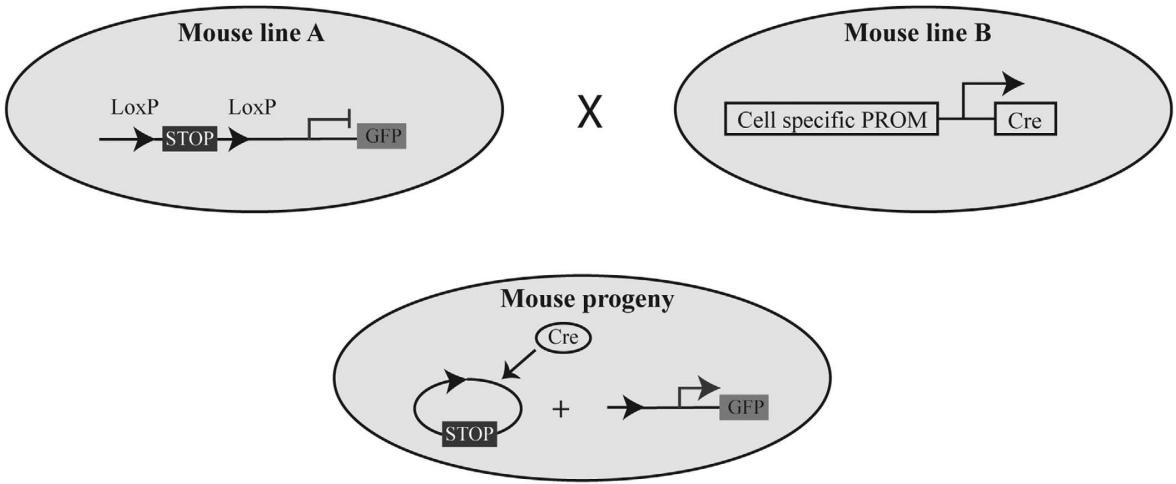

Figure 2 Schematic description of the lineage-tracing system. In the genetic lineage-tracing Cre-lox $P$ systems, the Cre recombinase expression is under the control of a tissue-/cell-specific promoter in one mouse line. This line is then crossed with a second one where the reporter gene is flanked by loxP-STOPloxP sequences. If the progeny contains both the alleles, Cre recombinase will determine the expression of the reporter gene by excision of one of the IoxP site. A full colour version of this figure is available at the Immunology and Cell Biology journal online.

drive their progression. However, the future of cancer research relies on pushing these boundaries by integrating different optical imaging approaches, to better define in vivo tumour structures, activity and aggressiveness. Few of the possible approaches are described in the next paragraphs.

\section{Bioluminescence imaging}

Bioluminescence imaging (BLI) is a powerful tool that it is accelerating our knowledge on the mechanisms adopted by cancer cells in situ to proliferate and disseminate in their native surrounding microenvironment. This imaging modality relies on luciferase reporter systems, with some technical advantages compared to fluorescent imaging. First of all, BLI reduces signal background, as cells do not emit light; the system and the instrumentation are user-friendly, and with limited costs; furthermore, luciferase enzymes have a very short half-life compared to fluorescent proteins, and this is useful to reliably analyse in vivo transcriptional activation, protein-protein interaction and other rapid biological processes. ${ }^{20}$ Therefore, in recent years a multitude of genetically engineered mouse models carrying luciferase reporters have been developed to study the molecular events occurring during cancer development and progression.

Spiotto et al. ${ }^{21}$ developed a luciferase reporter system to study the effect of tumour microenvironment on tumour unfolded protein response (UPR) and endoplasmic reticulum stress. Specifically, they created a transgenic mouse where the luciferase gene was under the control of the UPR component XBP-1 (XBP1-Luc transgene). In a spontaneous model of breast cancer, they were able to demonstrate that luciferase luminescence was correlated with tumour growth, thus suggesting that alteration of UPR pathway and endoplasmic reticulum stress were associated with tumour growth. Thus, UPR is currently considered as an emerging target for anticancer therapies. ${ }^{22}$ In addition, this study opened to the possibility to use BLI to define new markers and parameters for tumour grading and response to chemotherapy.

Goldman et al..$^{23}$ developed a luciferase reporter system to monitor hypoxia in mammary tumours. Thus luciferase expression, under the control of Hif- $1 \alpha$ (Hypoxic RE $>$ ODD-Luc) - the well-known marker of the hypoxic state within a tissue, provides a powerful means to monitor not only tumour growth but also simultaneously tumour hypoxia in a non-invasive manner.
Similar to fluorescence approaches, BLI can be used also to characterise the role of specific oncogenes in solid tumours. For instance, combining mouse models with luciferase expression driven by $\beta$-actin promoter in tandem with $\mathrm{Kras}^{\mathrm{v} 12}$ (constitutive activation of oncogenic Kras), provides a means to activate spontaneous lung tumours by adeno-Cre inhalation. Importantly, the spontaneous lung tumours developed in these mice can be monitored for up to 100 days after formation in a non-invasive manner, ${ }^{24}$ thus providing a wealth information on the temporal and spatial interaction of primary and secondary oncogenes. The exent of this temporal resolution is highlighted by prostate cancer models that can be traced for up to 400 days. ${ }^{25}$ The literature described above represents only few examples of the use of BLI to study tumour progression in vivo, as this imaging modality has been successfully established also in other tumour models, including pancreas and cervical cancer. ${ }^{26,27}$

Finally, BLI modality has been recently combined with fluorescent reporters to monitor in vivo and ex vivo breast cancer initiation and spontaneous dissemination. In particular, the authors created a dual Luc2-eGFP reporter to assess CD $44^{+}$breast CSCs cellular behaviours in orthotopic mouse models. The sensitivity of this approach is highlighted by the observation that the authors could detect as low as 10 injected CD $44^{+}$CSCs, and trace their involvement in spontaneous lung metastasis. Thus, the authors provided for the first time a tumour model system-combined with non-invasive imaging modality-that could be used to study the molecular events involved in the dissemination of CSCs. ${ }^{28}$

This approach is very straightforward, and allows a thorough analysis of tumour dissemination. Although still in infancy, combining BLI and fluorescence imaging stands as a powerful tool for understanding CSC, metastasis and the involvement of the microenvironment in tumorigenesis.

\section{Intravital microscopy to study cancer initiation and tumour cell/microenvironment interaction}

Live cell in vivo imaging, or intravital microscopy, is a technique that was recently developed to follow in real-time cellular behaviours in an unperturbed tissue, in either physiological or disease states. The advancement of intravital imaging has recently progressed thanks to the employment of the multiphoton laser scanner microscopy. This approach allows deep penetration within living tissues (up to $1 \mathrm{~mm}$ ), 
without side effects such as phototoxicity. In addition, it avoids or in some cases reduces the autofluorescence signal, thus enabling real-time imaging without interferences that can mislead the interpretation of the results. Intravital imaging is a powerful tool that can be used to address questions that would be impossible to answer by static analysis. For instance, Valentina Greco's group has extensively applied live imaging to define stem cell behaviour during skin regeneration. In particular, by combining elegant mouse reporter systems, they provided new insights on how skin stem cells regulated tissue regeneration in physiological conditions. ${ }^{29}$

In the past years, intravital imaging was used to study mechanisms of tumorigenesis. For example, it defined (1) the early events occurring during tumour development and (2) the mechanisms of tumour cell/microenvironment interaction, leading to cancer cell metastasis.

Recently, by combining intravital imaging with zebrafish models of melanoma (in the context of p53 and BRAF mutations), Kauffman et al. ${ }^{30}$ have demonstrated that the initial step leading to tumour formation was the transformation of melanocytes cells into embryonic progenitor-like cells. Interestingly, these findings supported previous results obtained by Blanpain's group in a murine model of basal cell carcinomas. $^{31}$

Intravital imaging has also contributed in determining how a small number of mutated cells can generate a heterogeneous tumour. By using a genetic mouse model system where few skin epithelial stem cells constitutively activate Wnt signalling, Deschene et al. ${ }^{32}$ demonstrated that these cells recruited wild-type neighbouring cells into the developing tumour. Although this finding was critical in understanding the biological processes of cancer initiation, therapeutic approaches targeting the wild-type cells of the tumours are more complex in design; thus, important efforts are needed to define treatments that can arrest cell recruitment in the tumour mass. Next-generation intravital imaging required simultaneous analysis of the microenvironment itself. These type of studies required additional tools labelling the tumour niche as well. For instance, endothelial cells or macrophages can be labelled with fluorescein isothiocyanate (FITC)-dextran or quantum dots. ${ }^{33}$ The extracellular matrix, and in particular the collagen, can be visualised by multiphoton laser scanner microscopy with the second harmonic signal. ${ }^{34}$ By using these approaches, several groups were able to elucidate the cellular mechanisms by which cancer cells invade the surrounding stroma, and colonise other organs (reviewed in depth by Ellenbroek and Rheenen $^{35}$ ).

Overall, intravital imaging is rapidly contributing to increase our understanding of how cells can form a tumour, and how they escape to colonise new organs. The next frontier is translating these imaging approaches for therapeutic purposes. In particular, it will be important to set up appropriate tools to follow molecules in real time as well as therapeutic compounds and their specific targets within the tissues. This approach has been successfully established in ex vivo cancer explants, ${ }^{36}$ thus setting the scene for troubleshooting the next generation of live imaging in clinical setting.

\section{CLINICAL IMAGING}

Imaging represents a critical part in the diagnosis, prognosis and follow-up of patients with cancer. Recent advances in clinical oncology have led to the development of new imaging methods able to (1) detect initial molecular and cellular events that determine the onset and progression of the oncological disease in the individual, (2) display the expression of molecular targets to design individualised therapies, (3) follow in real time the natural evolution of the disease and (4) monitor the efficacy of the therapy.

Up to now, diagnostic imaging procedures in cancer are largely applied in the management of symptomatic individuals; efforts are being made to improve these techniques for early detection in asymptomatic patients. Traditional diagnostic imaging procedures consist of MRI; X-ray computed tomography (CT) and ultrasound. Through these approaches, it is possible to obtain anatomical pictures, and therefore consider only tumour volume and tissue density as measure of the disease.

\section{Clinical Imaging}

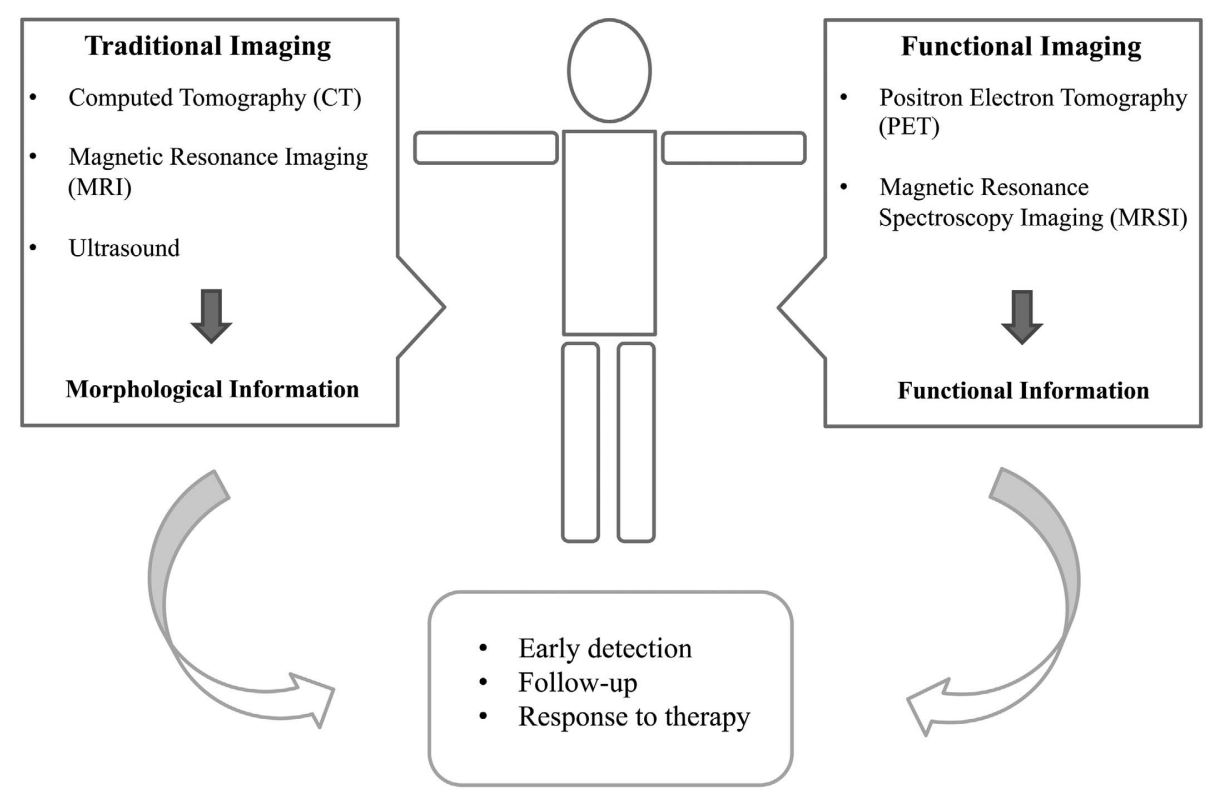

Figure 3 Schematic representation of imaging approaches in clinical oncology. A full colour version of this figure is available at the Immunology and Cell Biology journal online. 


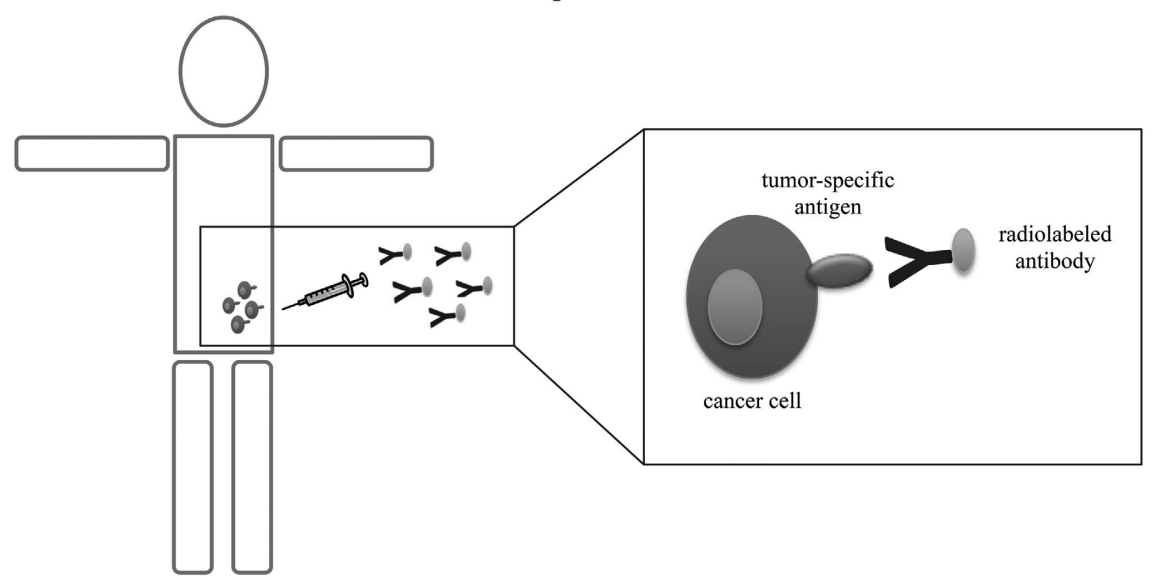

Figure 4 Schematic illustration of theranostic application for targeted therapy. A full colour version of this figure is available at the Immunology and Cell Biology journal online.

However, since the physiological or functional changes often precede anatomical modifications, these techniques fail to characterise the tumour; therefore the analysis of the function becomes a key component in the identification of the pathological process in play. A more recent advantage of imaging procedures consists of the introduction of molecular imaging that provides specific information on tissue metabolic states, by combining morphological information of CT with metabolic data. ${ }^{37,38}$ Amongst the high-resolution functional imaging techniques are PET and PET coupled with CT (PET/CT) and magnetic resonance spectroscopy (MRSI). By measuring chemical and biological processes for the detection of the cellular changes that occur early in the course of disease, molecular imaging techniques give information before the structural changes occur. And it is for these reasons that these approaches represent essential future tools in clinical oncology (Figure 3).

Each technology has some compromise on sensitivity, temporal resolution, spatial resolution, depth of signal, signal source and the availability of biocompatible probes.

In this section, we will briefly describe imaging approaches focusing on their clinical application in oncological patients.

\section{Positron emission tomography (PET) and combined diagnostic techniques: PET/CT, SPECT/CT}

$\mathrm{PET}$ and combination PET/CT are routinely used approaches in cancer diagnosis and treatment monitoring. PET is a diagnostic imaging technique that allows to measure chemical and biological processes that occur in the body. PET uses radioactive tracers obtained by marking molecules normally present in biological tissues (such as sugars, amino acids, water and so on) with positron emitting radionuclides $\beta+$ (particles with the same mass of electrons, but with a positive electrical charge).

PET analysis evaluates physiological and biochemical features in addition to morphological ones. For these reasons, it provides a biological characterisation for early and accurate detection. General indication for applying PET in oncology are (1) the differential diagnosis between benign and malignant lesions, (2) staging and diagnosis of relapse in order to adopt the best treatment and (3) monitoring the response to therapy.

The advantage of PET in oncology is the possibility of total-body evaluation and the biological characterisation of the tumour. Indeed this approach provides information on tumour perfusion, hypoxia, receptor expression, tumour metabolism and on the occurrence of other biological processes. PET involves the use of a radiotracer injected into the patient bloodstream. Since neoplastic transformation is associated to an increased glucose uptake and glycolysis, proportional to tumour growth and aggressiveness, one of the most used PET radiotracer is the 2-deoxy-2-[ $\left[{ }^{18} \mathrm{~F}\right]$-fluoro-D-glucose $\left({ }^{18} \mathrm{~F}-\mathrm{FDG}\right),{ }^{39}$ an analogue of glucose. Images obtained after injection of FDG show the areas of increased glucose metabolism thus detecting the localisation of primary tumour and of any metastases. The application of ${ }^{18} \mathrm{~F}$-FDG PET is more sensitive and specific in some cancers, for example, non-small cell lung cancer, ${ }^{40}$ melanoma, ${ }^{41}$ colorectal carcinoma, ${ }^{42}$ breast cancer ${ }^{43}$ head and neck tumours ${ }^{44}$ while poor ${ }^{18} \mathrm{~F}-\mathrm{FDG}$ uptake has been observed in prostate cancer, hepatocellular carcinoma, in small and non-invasive tumours.

In brain oncology, PET with ${ }^{18} \mathrm{~F}$-FDG is able to differentiate between low and high degree of malignancy and is useful in the differential diagnosis between tumour recurrence and fibrosis observed after treatment. ${ }^{45}$ Lung cancer can be recognised with conventional diagnostic imaging techniques, which, however, have limited diagnostic accuracy. It has been shown that PET studies identify malignant lesions in $96 \%$ of cases. ${ }^{46}$

Among other radiotracers used in oncological application of PET there are $\left[{ }^{18} \mathrm{~F}\right] \mathrm{L}-2$-Fluorothyrosine and ${ }^{11} \mathrm{C}$-Metionin, indicators of protein metabolism, and molecules as $\left[{ }^{18} \mathrm{~F}\right] \mathrm{FAZA},{ }^{47}\left[{ }^{18} \mathrm{~F}\right] \mathrm{MISO},{ }^{48}$ $\left[{ }^{62} \mathrm{Cu}\right] \mathrm{ATSM}$, used to evaluate tissue hypoxia.

The different imaging techniques often provide diverse morphological or functional information. They differ in the level of invasiveness, cost, quality of the acquired images and spatial or temporal resolution. Each method presents peculiarities and limitations; therefore, a combined use of biomedical imaging methods represents an effective approach.

The PET/CT is one of the most innovative diagnostic tools. It allows the detection of disease extension and localisation to arrange appropriate treatment plans optimised to selectively irradiate the metabolically active tumour tissues. The PET/CT combination provides better results than PET or CT alone, as it offers a more precise localisation of radiotracer, and distinguishes the physical uptake from the pathological one. Therefore, the significant advantages of PET/CT imaging is the accuracy of diagnosis. ${ }^{49}$

The single-photon emission computed tomography (SPECT) consists of the injection of a radiotracer in the patient and in the 
subsequent detection of gamma photons released from affected tissues. ${ }^{50}$ An advantage of SPECT is the improvement of the contrast in the region of interest compared to the background. The use of hybrid SPECT/CT is a more accurate method to combine the functional information provided by SPECT with the anatomical images provided by CT images. ${ }^{51}$

Despite the tremendous advantages in the diagnosis and follow-up of oncological patients, nuclear radiation used in PET, and in the combined techniques, precludes its repetitive use.

\section{Magnetic resonance imaging (MRI) and magnetic resonance spectroscopy imaging (MRSI)}

MRI is a modern diagnostic technique that provides detailed anatomical images of the human body; in particular, taking advantage of the magnetic and electric capacity of the electron, MRI allows the analysis of biological tissues through the evaluation of electron energy absorption.

With this technique, many diseases or alterations relative to internal organs can be displayed and diagnosed. MRI can be used for the diagnosis of a wide variety of pathological conditions and is particularly useful in the diagnosis of diseases of the brain and spine, abdomen and pelvis, of the great vessels and of the musculoskeletal system. The main advantages of MRI are high contrast resolution and the absence of ionising radiation. Indeed the key feature of MRI is to employ radiation with low electromagnetic energy content that does not modify or destroy biological molecules.

MRI generates anatomic data, without considering organ functionality. MRSI is an imaging technique that provides metabolic information of normal or pathological tissues; in oncological application, MRSI shows not only biochemical changes in response to tumour growth but also delineates different metabolic tumour phenotypes, without the use of radiotracer..$^{52}$

Among the active nuclei used to monitor the metabolic changes that occur in cancer tissue there are proton MRSI ( ${ }^{1} \mathrm{H}$ MRS), ${ }^{31} \mathrm{P}$ (phosphorus), ${ }^{13} \mathrm{C}$ (carbon) and ${ }^{19} \mathrm{~F}$ (fluorine). To date, proton MRSI is the method most commonly used for clinical purposes ${ }^{53}$ because it allows to obtain high-resolution spectra from small volumes of interest and to identify numerous metabolites with different biochemical meaning.

In clinical practice, MRSI is particularly useful in the diagnosis of brain tumours. ${ }^{54}$ Choline ratios with other metabolites are routinely used to classify cancer aggressiveness; ${ }^{55}$ indeed, by monitoring the ratio cholin/creatin, is possible to obtain a spectroscopic map that identify the neoplastic activity in each site. Proton MRI allows the non-invasive diagnosis of prostate cancer. ${ }^{56}$ The molecular markers of this tumour are citrate, creatin and choline; therefore, changes of metabolites predict with accuracy the presence of tumour. In addition, ${ }^{1} \mathrm{H}$ MRS allows the diagnosis of breast cancer in relation to the presence of choline and the ratio of the choline/creatin. ${ }^{57}$ Proton MRSI also provides early information on treatment responses, discriminating responders to not-responders.

${ }^{31} \mathrm{P}$ MRS detects metabolites such as phosphocreatine, nucleotide triphosphate, phosphomonoesters (phosphocholine, phosphoethanolamine and phosphoserine), phosphodiesters (glycerylphosphocholine, glycerophosphoethanolamine and glycerylphosphoserine) and inorganic phosphate $(\mathrm{Pi})$. Through this approach, it is possible to determine intracellular $\mathrm{pH}$, glycolysis and oxidative phosphorylation. Compared to the proton spectroscopy, the phosphor is characterised by a low sensitivity and by a lower spatial resolution, but is able to provide a complete representation of energy flows in biological systems. Phosphorus MRI has been mainly applied in the diagnosis and follow-up of breast cancer, ${ }^{58}$ where phosphomonoesters levels is increased.

\section{Imaging for targeted therapy}

Progresses in imaging techniques have been fundamental in biomedical sciences; nowadays, imaging not only represents a crucial tool for monitoring treatment efficacy but also it is considered an important approach for several types of cancer treatment. Novel treatments combine the role of imaging probes with therapeutic applications. The primary goal is to deliver the therapy directly to the tumour site with minimal systemic toxicity.

Recent advantages in cell biology as well as molecular imaging highlight the possibility to develop a new class of compounds, called theranostic compounds, that combine molecular imaging approaches to therapeutic ones (Figure 4).

It is well established that cancer tissues overexpress specific proteins that serve as target for designed therapy. For example, targeted radionuclide therapy is based on the use of molecules, with highspecificity for target cells, as carriers of radionuclides directly to tumour and is now emerging as an effective therapeutic option for several cancer types. ${ }^{59}$

One of the most suitable tumour types for radionuclide therapy is prostate cancer where prostate-specific membrane antigen is highly overexpressed. Some clinical trials have showed promising data on the application of the monoclonal antibody J591, to prostate-specific membrane antigen, radiolabelled with $90 \mathrm{Y}$ or $177 \mathrm{Lu}$ in castrationresistant prostate cancer patients. ${ }^{59}$ In addition to prostate cancer, also melanoma can be targeted with radionuclide therapy. ${ }^{60}$ Although very promising, some challenges need to be overcome for the routine application of theranostics in clinical oncology; a main problem is indeed associated with the high doses of ionising radiation needed for tumour tissue destruction.

\section{CONCLUDING REMARKS}

Recent years have seen tremendous advances in cancer research and management as a result of (1) a better understanding of the biological processes adopted by cancer cells to initiate tumours and colonise secondary metastatic sites, (2) innovative approaches for cancer early detection and (3) defining new and personalised therapeutical strategies aiming at the eradication of the disease. Next generation of biomedical imaging modalities, and in particular of molecular imaging, have provided key biological information that would have been impossible to obtain by standard approaches. While many questions still need to be addressed, the discovery of new and accurate biocompatible tracers as well as the development of new higher imaging resolution and contrast, have the potential to revolutionise our understanding of basic cancer cell biology and its treatment.

\section{CONFLICT OF INTEREST}

The authors declare no conflict of interest.

\section{ACKNOWLEDGEMENTS}

We kindly thank Prof Riccardo Alessandro (University of Palermo) for critical suggestions on the manuscript. SR is supported by a 'FIRC' (Fondazione Italiana Ricerca sul Cancro) fellowship.

1 Siegel R, Ma J, Zou Z, Jemal A. Cancer statistics, 2014. CA Cancer J Clin 2014; 64: 9-29.

2 Jemal A, Siegel R, Ward E, Hao Y, Xu J, Thun MJ. Cancer statistics, 2009. CA Cancer J Clin 2009; 59: 225-249. 
3 Al-Hajj M, Becker MW, Wicha M, Weissman I, Clarke MF. Therapeutic implications of cancer stem cells. Curr Opin Genet Dev 2004; 14: 43-47.

4 Balkwill FR, Capasso M, Hagemann T. The tumor microenvironment at a glance. J Cell Sci 2012; 125: 5591-5596.

5 Weissleder R, Nahrendorf M. Advancing biomedical imaging. Proc Natl Acad Sci USA 2015; 112: 14424-14428.

6 Hart LS, El-Deiry WS. Invincible, but not invisible: imaging approaches toward in vivo detection of cancer stem cells. J Clin Oncol 2008; 26: 2901-2910.

7 Collins AT, Berry PA, Hyde C, Stower MJ, Maitland NJ. Prospective identification of tumorigenic prostate cancer stem cells. Cancer Res 2005; 65: 10946-10951.

8 Vlashi E, Kim K, Lagadec C, Donna LD, McDonald JT, Eghbali M et al. In vivo imaging, tracking, and targeting of cancer stem cells. J Nat/ Cancer Inst 2009; 101: 350-359.

9 Soriano P. Generalized lacZ expression with the ROSA26 Cre reporter strain. Nat Genet 1999; 21: 70-71.

10 Mao X, Fujiwara Y, Chapdelaine A, Yang H, Orkin SH. Activation of EGFP expression by Cre-mediated excision in a new ROSA26 reporter mouse strain. Blood 2001; 97 324-326.

11 Alcolea MP, Jones PH. Tracking cells in their native habitat: lineage tracing in epithelial neoplasia. Nat Rev Cancer 2013; 13: 161-171.

12 Blanpain C. Tracing the cellular origin of cancer. Nat Cell Biol 2013; 15: 126-134.

13 Youssef KK, Van Keymeulen A, Lapouge G, Beck B, Michaux C, Achouri Y et al. Identification of the cell lineage at the origin of basal cell carcinoma. Nat Cell Biol 2010; 12: 299-305.

14 White AC, Tran K, Khuu J, Dang C, Cui Y, Binder SW et al. Defining the origins of Ras/p53-mediated squamous cell carcinoma. Proc Natl Acad Sci USA 2011; 108 7425-7430.

15 Lapouge G, Youssef KK, Vokaer B, Achouri Y, Michaux C, Sotiropoulou PA et al. Identifying the cellular origin of squamous skin tumors. Proc Natl Acad Sci USA 2011; 108: 7431-7436.

16 Zito G, Saotome I, Liu Z, Ferro EG, Sun TY, Nguyen DX et al. Spontaneous tumour regression in keratoacanthomas is driven by Wnt/retinoic acid signalling cross-talk. Nat Commun 2014; 5: 3543

17 Sutherland KD, Proost N, Brouns I, Adriaensen D, Song JY, Berns A. Cell of origin of small cell lung cancer: inactivation of Trp53 and Rb1 in distinct cell types of adult mouse lung. Cancer Cell 2011; 19: 754-764.

18 Barker N, Ridgway RA, van Es JH, van de Wetering $\mathrm{M}$, Begthel $\mathrm{H}$, van den Born et al. Crypt stem cells as the cells-of-origin of intestinal cancer. Nature 2009; 457: 608-611.

19 Schepers AG, Snippert HJ, Stange DE, van den Born M, van Es JH, van de Wetering M et al. Lineage tracing reveals $\mathrm{Lgr}^{+}$stem cell activity in mouse intestinal adenomas. Science 2012; 337: 730-735.

20 Kocher B, Piwnica-Worms D. Illuminating cancer systems with genetically engineered mouse models and coupled luciferase reporters in vivo. Cancer Discov 2013; 3: 616-629.

21 Spiotto MT, Banh A, Papandreou I, Cao H, Galvez MG, Gurtner GC et al. Imaging the unfolded protein response in primary tumors reveals microenvironments with metabolic variations that predict tumor growth. Cancer Res 2010; 70: 78-88.

22 Moenner M, Pluquet O, Bouchecareilh M, Chevet E. Integrated endoplasmic reticulum stress responses in cancer. Cancer Res 2007; 67: 10631-10634.

23 Goldman SJ, Chen E, Taylor R, Zhang S, Petrosky W, Reiss M et al. Use of the ODD-luciferase transgene for the non-invasive imaging of spontaneous tumors in mice. PLOS ONE 2011; 6: e18269.

24 Lyons SK, Meuwissen R, Krimpenfort P, Berns A. The generation of a conditional reporter that enables bioluminescence imaging of Cre/loxP-dependent tumorigenesis in mice. Cancer Res 2003; 63: 7042-7046.

25 Liao CP, Zhong C, Saribekyan G, Bading J, Park R, Conti PS et al. Mouse models of prostate adenocarcinoma with the capacity to monitor spontaneous carcinogenesis by bioluminescence or fluorescence. Cancer Res 2007; 67: 7525-7533.

26 Zumsteg A, Strittmatter K, Klewe-Nebenius D, Antoniadis H, Christofori G. A bioluminescent mouse model of pancreatic \{beta\}-cell carcinogenesis. Carcinogenesis 2010; 31: 1465-1474

27 Jabbar SF, Abrams L, Glick A, Lambert PF. Persistence of high-grade cervical dysplasia and cervical cancer requires the continuous expression of the human papillomavirus type 16 E7 oncogene. Cancer Res 2009; 69: 4407-4414.

28 Liu H, Patel MR, Prescher JA, Patsialou A, Qian D, Lin J et al. Cancer stem cells from human breast tumors are involved in spontaneous metastases in orthotopic mouse models. Proc Natl Acad Sci USA 2010; 107: 18115-18120.

29 Rompolas P, Deschene ER, Zito G, Gonzalez DG, Saotome I, Haberman AM et al. Live imaging of stem cell and progeny behaviour in physiological hair-follicle regeneration. Nature 2012; 487: 496-499.

30 Kaufman CK, Mosimann C, Fan ZP, Yang S, Thomas AJ, Ablain J et al. A zebrafish melanoma model reveals emergence of neural crest identity during melanoma initiation. Science 2016; 351: aad2197.

31 Youssef KK, Lapouge G, Bouvree K, Rorive S, Brohee S, Appelstein 0 et al. Adult interfollicular tumour-initiating cells are reprogrammed into an embryonic hair follicle progenitor-like fate during basal cell carcinoma initiation. Nat Cell Biol 2012; 14: 1282-1294.
32 Deschene ER, Myung P, Rompolas P, Zito G, Sun TY, Taketo MM et al. beta-Catenin activation regulates tissue growth non-cell autonomously in the hair stem cell niche. Science 2014; 343: 1353-1356.

33 Wyckoff JB, Pinner SE, Gschmeissner S, Condeelis JS, Sahai E. ROCK- and myosin-dependent matrix deformation enables protease-independent tumor-cell invasion in vivo. Curr Biol 2006; 16: 1515-1523.

34 Friedl P, Wolf K, von Andrian UH, Harms G. Biological second and third harmonic generation microscopy. Curr Protoc Cell Biol 2007; Chapter 4: Unit 415.

35 Ellenbroek SI, van Rheenen J. Imaging hallmarks of cancer in living mice. Nat Rev Cancer 2014; 14: 406-418.

36 Raimondo S, Naselli F, Fontana S, Monteleone F, Lo Dico A, Saieva L et al. Citrus limon-derived nanovesicles inhibit cancer cell proliferation and suppress CML xenograft growth by inducing TRAIL-mediated cell death. Oncotarget 2015; 6: 19514-19527.

37 Beyer T, Townsend DW, Blodgett TM. Dual-modality PET/CT tomography for clinical oncology. Q J Nucl Med 2002; 46: 24-34.

38 Avril S, Muzic RF Jr., Plecha D, Traughber BJ, Vinayak S, Avril N. (1)(8)F-FDG PET/CT for monitoring of treatment response in breast cancer. J Nucl Med 2016; 57: 34S-39S.

39 Almuhaideb A, Papathanasiou N, Bomanji J. 18F-FDG PET/CT imaging in oncology. Ann Saudi Med 2011; 31: 3-13.

40 Soussan M, Cyrta J, Pouliquen C, Chouahnia K, Orlhac F, Martinod E et al. Fluorine 18 fluorodeoxyglucose PET/CT volume-based indices in locally advanced non-small cell lung cancer: prediction of residual viable tumor after induction chemotherapy. Radiology 2014; 272: 875-884.

41 Fuster D, Chiang S, Johnson G, Schuchter LM, Zhuang H, Alavi A. Is 18F-FDG PET more accurate than standard diagnostic procedures in the detection of suspected recurrent melanoma? J Nucl Med 2004; 45: 1323-1327.

42 Llamas-Elvira JM, Rodriguez-Fernandez A, Gutierrez-Sainz J, Gomez-Rio M, Bellon-Guardia M, Ramos-Font $\mathrm{C}$ et al. Fluorine-18 fluorodeoxyglucose PET in the preoperative staging of colorectal cancer. Eur J Nucl Med Mol Imaging 2007; 34: 859-867.

43 Liu H, Chen Y, Wu S, Song F, Zhang H, Tian M. Molecular imaging using PET and SPECT for identification of breast cancer subtypes. Nucl Med Commun 2016; 37: 1116-1124.

44 Sheikhbahaei S, Marcus C, Subramaniam RM. 18F FDG PET/CT and head and neck cancer: patient management and outcomes. PET Clin 2015; 10: 125-145.

45 Padma MV, Said S, Jacobs M, Hwang DR, Dunigan K, Satter M et al. Prediction of pathology and survival by FDG PET in gliomas. J Neurooncol 2003; 64: 227-237.

46 Fischer BM, Mortensen J, Hojgaard L. Positron emission tomography in the diagnosis and staging of lung cancer: a systematic, quantitative review. Lancet Oncol 2001; 2: 659-666.

47 Trinkaus ME, Blum R, Rischin D, Callahan J, Bressel M, Segard T et al. Imaging of hypoxia with 18F-FAZA PET in patients with locally advanced non-small cell lung cancer treated with definitive chemoradiotherapy. J Med Imaging Radiat Oncol 2013; 57: 475-481.

48 Hirata K, Terasaka S, Shiga T, Hattori N, Magota K, Kobayashi H et al. 1)(8)F-Fluoromisonidazole positron emission tomography may differentiate glioblastoma multiforme from less malignant gliomas. Eur J Nucl Med Mol Imaging 2012; 39: 760-770.

49 Griffeth LK. Use of PET/CT scanning in cancer patients: technical and practical considerations. Proc (Bayl Univ Med Cent) 2005; 18: 321-330.

50 Cai J, Li F. Single-photon emission computed tomography tracers for predicting and monitoring cancer therapy. Curr Pharm Biotechnol 2013; 14: 693-707.

51 Schillaci O. Single-photon emission computed tomography/computed tomography in lung cancer and malignant lymphoma. Semin Nucl Med 2006; 36: 275-285.

52 Haris M, Yadav SK, Rizwan A, Singh A, Wang E, Hariharan H et al. Molecular magnetic resonance imaging in cancer. J Trans/ Med 2015; 13: 313.

53 Kwock L, Smith JK, Castillo M, Ewend MG, Collichio F, Morris DE et al. Clinical role of proton magnetic resonance spectroscopy in oncology: brain, breast, and prostate cancer. Lancet Oncol 2006; 7: 859-868.

54 Barker PB. N-acetyl aspartate-a neuronal marker? Ann Neurol 2001; 49: 423-424.

55 Bolan PJ, Meisamy S, Baker EH, Lin J, Emory T, Nelson M et al. In vivo quantification of choline compounds in the breast with 1 H MR spectroscopy. Magn Reson Med 2003; 50: $1134-1143$

56 Kurhanewicz J, Swanson MG, Nelson SJ, Vigneron DB. Combined magnetic resonance imaging and spectroscopic imaging approach to molecular imaging of prostate cancer. J Magn Reson Imaging 2002; 16: 451-463.

57 Klomp DW, van de Bank BL, Raaijmakers A, Korteweg MA, Possanzini C, Boer VO et al. 31P MRSI and $1 \mathrm{H}$ MRS at $7 \mathrm{~T}$ : initial results in human breast cancer. NMR Biomed 2011; 24: 1337-1342.

58 Park JM, Park JH. Human in-vivo 31P MR spectroscopy of benign and malignant breast tumors. Korean J Radiol 2001; 2: 80-86.

59 Tagawa ST, Milowsky MI, Morris M, Vallabhajosula S, Christos P, Akhtar NH et al. Phase II study of Lutetium-177-labeled anti-prostate-specific membrane antigen monoclonal antibody J591 for metastatic castration-resistant prostate cancer. Clin Cancer Res 2013; 19: 5182-5191.

60 Allen BJ, Singla AA, Rizvi SM, Graham P, Bruchertseifer F, Apostolidis C et al. Analysis of patient survival in a Phase I trial of systemic targeted alpha-therapy for metastatic melanoma. Immunotherapy 2011; 3: 1041-1050. 